\title{
The High Time Resolution Universe Pulsar Survey - XIII. PSR J1757-1854, the most accelerated binary pulsar
}

\author{
A. D. Cameron ${ }^{1 \star}$, D. J. Champion ${ }^{1}$, M. Kramer ${ }^{1,2}$, M. Bailes $^{3,4,5}$, E. D. Barr ${ }^{1}$,
} C. G. Bassa ${ }^{6}$, S. Bhandari ${ }^{3,4}$, N. D. R. Bhat ${ }^{4,7}$, M. Burgay $^{8}$, S. Burke-Spolaor ${ }^{9,10}$, R. P. Eatough ${ }^{1}$, C. M. L. Flynn ${ }^{3}$, P. C. C. Freire ${ }^{1}$, A. Jameson ${ }^{3,4}$, S. Johnston ${ }^{11}$, R. Karuppusamy ${ }^{1}$, M. J. Keith ${ }^{2}$, L. Levin ${ }^{2}$, D .R. Lorimer ${ }^{9}$, A. G. Lyne ${ }^{2}$, M. A. McLaughlin ${ }^{9}$, C. Ng ${ }^{12}$, E. Petroff ${ }^{6}$, A. Possenti ${ }^{8}$, A. Ridolfi ${ }^{1}$, B. W. Stappers ${ }^{2}$, W. van Straten ${ }^{3,4,13}$, T. M. Tauris ${ }^{1,14}$, C. Tiburzi ${ }^{1,15}$, N. Wex ${ }^{1}$

${ }_{1}^{1}$ Max-Planck-Institut für Radioastronomie, Auf dem Hügel 69, D-53121 Bonn, Germany.

2 Jodrell Bank Center for Astrophysics, University of Manchester, Alan Turing Building, Oxford Road, Manchester M13 9PL, United Kingdom.

${ }^{3}$ Centre for Astrophysics and Supercomputing, Swinburne University of Technology, Mail H39, PO Box 218, VIC 3122, Australia.

${ }^{4}$ ARC Center of Excellence for All-Sky Astronomy (CAASTRO), Swinburne University of Technology, Mail H30, PO Box 218, VIC 3122, Austral

${ }^{5}$ ARC Center of Excellence for Gravitational Wave Discovery (OzGrav), Swinburne University of Technology, Mail H11, PO Box 218, VIC 3122,

${ }^{6}$ ASTRON, the Netherlands Institute for Radio Astronomy, Postbus 2, NL-7990 AA Dwingeloo, the Netherlands.

${ }^{7}$ International Centre for Radio Astronomy Research, Curtin University, Bentley, WA 6102, Australia.

${ }^{8}$ INAF - Osservatorio Astronomico di Cagliari, Via della Scienza 5, I-09047 Selargius (CA), Italy.

${ }^{9}$ Department of Physics and Astronomy, West Virginia University, PO Box 6315, Morgantown, WV 26506, USA.

${ }^{10}$ Center for Gravitational Waves and Cosmology, West Virginia University, Chestnut Ridge Research Building, Morgantown, WV 26505, USA.

${ }^{11}$ CSIRO Astronomy \&5 Space Science, Australia Telescope National Facility, P.O. Box 76, Epping, NSW 1710, Australia.

${ }^{12}$ Department of Physics and Astronomy, University of British Columbia, 6224 Agricultural Road, Vancouver, BC V6T 1Z1, Canada.

${ }^{13}$ Institute for Radio Astronomy \&6 Space Research, Auckland University of Technology, Private Bag 92006, Auckland 1142, New Zealand.

${ }^{14}$ Argelander-Insitut für Astronomie, Universität Bonn, Auf dem Hügel 71, 53121 Bonn, Germany

${ }^{15}$ Fakultät für Physik, Universität Bielefeld, Postfach 100131, D-33501 Bielefeld, Germany.

Accepted XXX. Received YYY; in original form ZZZ

\begin{abstract}
We report the discovery of PSR J1757-1854, a 21.5-ms pulsar in a highly-eccentric, 4.4-h orbit with a neutron star (NS) companion. PSR J1757-1854 exhibits some of the most extreme relativistic parameters of any known pulsar, including the strongest relativistic effects due to gravitational-wave (GW) damping, with a merger time of $76 \mathrm{Myr}$. Following a 1.6-yr timing campaign, we have measured five post-Keplerian $(\mathrm{PK})$ parameters, yielding the two component masses $\left(m_{\mathrm{p}}=1.3384(9) \mathrm{M}_{\odot}\right.$ and $m_{\mathrm{c}}=$ $1.3946(9) \mathrm{M}_{\odot}$ ) plus three tests of general relativity (GR), which the theory passes. The larger mass of the NS companion provides important clues regarding the binary formation of PSR J1757-1854. With simulations suggesting 3- $\sigma$ measurements of both the contribution of Lense-Thirring precession to the rate of change of the semi-major axis and the relativistic deformation of the orbit within $\sim 7-9$ years, PSR J1757-1854 stands out as a unique laboratory for new tests of gravitational theories.
\end{abstract}

Key words: pulsars: individual (PSR J1757-1854) - binaries: close - gravitation surveys

\section{INTRODUCTION}

Since the discovery of the Hulse-Taylor pulsar (PSR B1913+16; Hulse \& Taylor 1975), binary pul-

^ E-mail: acameron@mpifr-bonn.mpg.de sars have played a critical role in providing key tests of GR and its alternatives. Even with the direct detection of gravitational waves (Abbott et al. 2016) and the more recent direct observation of a double neutron star (DNS) merger (Abbott et al. 2017), binary pulsars are still able to provide some of the most important gravity tests with strongly

(C) 2017 The Authors 
self-gravitating bodies, particularly in the quasi-stationary strong-field gravity regime (Wex 2014). An example is the constraint on the leading-order GW emission in GR (as described by the quadrupole formula), for which the double pulsar (PSR J0737-3039; Burgay et al. 2003; Lyne et al. 2004) currently stands out as the most constraining system, surpassing LIGO by three orders of magnitude (Kramer 2016; Abbott et al. 2016). PSR J0737-3039 currently offers five independent tests of GR (based on six PK parameters and the mass ratio), of which it passes the most stringent to within a measurement uncertainty of only $0.05 \%$ (Kramer et al. 2006; Breton et al. 2008). Other binary pulsars, such as the millisecond pulsar-white dwarf PSR J1738+0333, provide strong constraints on dipolar GW emission, a prediction of many alternative theories of gravity such as scalar-tensor theories (Freire et al. 2012).

Pulsar constraints on the nature of GWs, the limits of GR and on alternative gravitational theories are anticipated to provide both complementary and competitive tests against the most advanced ground-based GW detectors currently foreseen (Shao et al. 2017). However, this depends upon the discovery of additional relativistic systems capable of expanding the parameter space currently explored by binary pulsars. To this end, the High Time Resolution Universe South Low Latitude pulsar survey (HTRU-S LowLat, Keith et al. 2010; Ng et al. 2015), conducted using the Parkes 64-m radio telescope, was undertaken with the specific goal of discovering additional relativistic binary pulsars. This survey covers the inner Galactic plane $\left(-80^{\circ}<l<30^{\circ}\right.$ and $\left.|b|<3.5^{\circ}\right)$ and is the region predicted to contain the highest number of relativistic binaries (Belczynski et al. 2002).

Here, we report the discovery of PSR J1757-1854, the first relativistic binary discovered in the HTRU-S LowLat survey. PSR J1757-1854 (see Fig. 1) is a 21.5-ms pulsar in a 4.4-h orbit with an eccentricity of 0.61 and a NS companion, making the system a DNS. The compactness, high eccentricity and short orbital period of PSR J1757-1854 make it one of the most relativistic binary pulsars known, with the potential for even more rigorous constraints to be placed on GR and other gravitational theories.

\section{DISCOVERY}

\subsection{Candidate identification and confirmation}

To search for binary pulsars, we employ the 'time-domain resampling' technique (see e.g. Middleditch \& Kristian 1984; Johnston \& Kulkarni 1991), which assumes that the binary motion can be modelled as a constant line-of-sight acceleration. For a circular orbit this assumption holds best when the quantity $r_{\mathrm{b}}=t_{\text {int }} / P_{\mathrm{b}} \leq 0.1$ where $t_{\text {int }}$ is the integration time of the observation and $P_{\mathrm{b}}$ is the orbital period (see e.g. Johnston \& Kulkarni 1991; Ng et al. 2015). Building on the technique of Eatough et al. (2013), our 'partially-coherent segmented acceleration search' uses this principle to blindly search for pulsars in compact binary systems by progressively halving each observation into smaller time segments (as low as $t_{\text {int }}=537 \mathrm{~s}$ ) which are independently searched (to accelerations as high as $|a|=1200 \mathrm{~m} \mathrm{~s}^{-2}$ ). This has the trade-off of increasing our sensitivity to shorter orbital periods while gradually reducing our sensitivity in flux density. Full details of the technique are available in $\mathrm{Ng}$ et al. (2015).

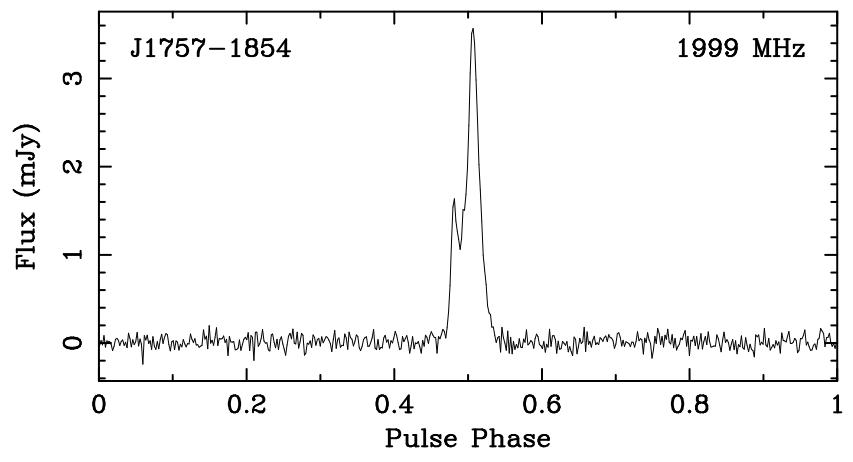

Figure 1. Pulse profile of PSR J1757-1854, observed with the Green Bank Telescope (GBT) on MJD 57857, integrated over approximately one full orbit.

PSR J1757-1854 was identified in the second 36-min half-length segment of a full 72-min observation recorded on MJD 56029, with an acceleration of $-32 \mathrm{~m} \mathrm{~s}^{-2}$ and a signal-to-noise ratio $(\mathrm{S} / \mathrm{N})$ of 13.3 . The pulsar's signal was recoverable across the full observation to a $\mathrm{S} / \mathrm{N}$ of 21.4 , but with a significantly-changing acceleration (i.e. 'jerk'). Consequently, a time-domain acceleration search of the full-length observation only detected the pulsar at a reduced $\mathrm{S} / \mathrm{N}$ of only 10.6, indicating that the segmented search greatly assisted in the discovery of this pulsar. At periastron, the pulsar reaches a maximum absolute acceleration of $\sim 684 \mathrm{~m} \mathrm{~s}^{-2}$, the highest of any known binary pulsar system.

\subsection{Non-detection in the PMPS}

The Parkes Multibeam Pulsar Survey (PMPS, Manchester et al. 2001) contains a beam coincident with the timed position of PSR J1757-1854 (see Table 2). However, neither acceleration searches using both time-domain resampling and matched filtering (Ransom et al. 2002) nor a fold using the ephemeris in Table 2 detected the pulsar in the PMPS data. Based upon the radiometer equation (see e.g. Lorimer \& Kramer 2004) and the dispersive smearing introduced by the $3-\mathrm{MHz}$ channel size of the PMPS, we expect a $\mathrm{S} / \mathrm{N}_{\mathrm{PMPS}} \simeq 8$, rendering any detection of PSR J1757-1854 difficult. Additional factors such as geodetic precession may also play a role, but at present a precise cause cannot be determined.

\section{TIMING}

\subsection{Observations and data reduction}

A summary of PSR J1757-1854's timing observations can be found in Table 1. Parkes (PKS) observations used the 21-cm Multibeam (MB20; Staveley-Smith et al. 1996) and $\mathrm{H}-\mathrm{OH}$ receivers, in combination with the Berkeley Parkes Swinburne Recorder ${ }^{1}$ (BPSR), the CASPER Parkes Swinburne Recorder ${ }^{2}$ (CASPSR) and a Digital Filter Bank system (DFB4). Jodrell Bank (JBO) observations with the 76-m Lovell telescope employed an L-Band receiver with a ROACH backend system (Bassa et al. 2016). Observations

$\begin{array}{ll}1 & \text { https://astronomy.swin.edu.au/pulsar/?topic=bpsr } \\ 2 & \text { https://astronomy.swin.edu.au/pulsar/?topic=caspsr }\end{array}$ 
Table 1. Telescope frontend and backend configurations, including the central frequency $\left(f_{\mathrm{c}}\right)$, bandwidth $(\Delta f)$ of each combination and the number $\left(n_{\text {TOA }}\right)$ and time span of the TOAs.

\begin{tabular}{|c|c|c|c|c|}
\hline Receiver & Backend & $\begin{array}{l}f_{\mathrm{c}}(\Delta f) \\
(\mathrm{MHz})\end{array}$ & $n_{\mathrm{TOA}}$ & $\begin{array}{l}\text { Span } \\
\text { (MJD) }\end{array}$ \\
\hline \multicolumn{5}{|l|}{$P K S:$} \\
\hline \multirow[t]{2}{*}{ MB20 } & BPSR & $1382(400)$ & 9 & $57405-57406$ \\
\hline & CASPSR $^{\mathrm{a}}$ & $1382(400)$ & 41 & $57734-57986$ \\
\hline \multirow[t]{2}{*}{$\mathrm{H}-\mathrm{OH}$} & DFB4 & $1369(256)$ & 57 & $57553-57675$ \\
\hline & CASPSR $^{\mathrm{a}}$ & $1382(400)$ & 55 & $57596-57635$ \\
\hline $\begin{array}{l}J B O: \\
\text { L-Band }\end{array}$ & \multicolumn{3}{|c|}{$J B O:$} & $57456-57958$ \\
\hline \multicolumn{5}{|l|}{$E F F:$} \\
\hline \multirow[t]{2}{*}{ 7-Beam } & FOLD & $1360(240)$ & 83 & $57573-57896$ \\
\hline & $\mathrm{BB}^{\mathrm{a}}$ & $1360(240)$ & 84 & $57815-57986$ \\
\hline \multicolumn{5}{|l|}{$G B T$ : } \\
\hline PF1-800 & GUPPI ${ }^{\mathrm{a}}$ & $820(200)$ & 25 & $57620-57621$ \\
\hline L-Band & GUPPI $^{\mathrm{a}, \mathrm{b}}$ & $1499(800)$ & 731 & $57795-57950$ \\
\hline S-Band & GUPPI $^{\mathrm{a}, \mathrm{b}}$ & $1999(800)$ & 1655 & $57627-57998$ \\
\hline
\end{tabular}

a Observations recorded with coherent de-dispersion.

b $\Delta f$ split into $200 \mathrm{MHz}$ sub-bands before TOA production.

at Effelsberg (EFF) were performed with the 7-Beam receiver with the PSRIX backend (Lazarus et al. 2016) operating in both a folded and baseband recording mode (FOLD and $\mathrm{BB}$ respectively). Finally, observations with the Green Bank Telescope (GBT) were conducted using the L-Band, S-Band and Prime-Focus 800-MHz (PF1-800) receivers, all in combination with the Green Bank Ultimate Pulsar Processing Instrument (GUPPI; DuPlain et al. 2008). All GBT and Effelsberg observations were designed to sample a full or significant fraction of the orbit.

Data reduction employed the DSPSR (van Straten \& Bailes 2011), PSRChive (Hotan et al. 2004), SIGPROC $^{3}$, PRESTO (Ransom 2001), TEMPO ${ }^{4}$ and TEMPO2 (Hobbs et al. 2006) software packages. Each TOA set was produced using its own reference profile (all rotated to the same pulse phase), and was weighted such that its reduced $\chi^{2}=1$. The data sets were combined using jumps fit across regions of common overlap. During this process, the pulsar's dispersion measure (DM) appeared to vary as a function of orbital phase. This resulted from an apparent inability of DSPSR and PSRCHIVE to correctly de-disperse across a large bandwidth during rapid orbital motion, despite this effect having been accounted for by TEMPO2's phase predictors (Hobbs et al. 2006). To counteract this, Jodrell Bank and GBT (L and S-Band) TOAs were produced from 200-MHz sub-bands.

\subsection{Measured parameters and implications}

Our derived ephemeris of PSR J1757-1854, employing the DDH (Freire \& Wex 2010) binary model, is provided in Table 2 . Based upon the spin parameters, we derive a characteristic age $\tau_{\mathrm{c}} \simeq 130 \mathrm{Myr}$ and a surface magnetic field $B_{\text {surf }} \simeq$

\footnotetext{
3 http://sigproc.sourceforge.net

4 http://tempo.sourceforge.net
}

Table 2. Ephemeris of PSR J1757-1854, as derived using TEMPO2. Numbers in parentheses represent $1-\sigma$ uncertainties, with TOA errors re-weighted such that the reduced $\chi^{2}$ went from 1.7 to 1.0. DM distances are derived from the NE2001 (Cordes \& Lazio 2002) and YMW16 (Yao et al. 2017) models.

\begin{tabular}{|c|c|}
\hline Right ascension, $\alpha$ (J2000) & $17: 57: 03.78438(6)$ \\
\hline Declination, $\delta(\mathrm{J} 2000)$ & $-18: 54: 03.376(7)$ \\
\hline Spin period, $P(\mathrm{~ms})$ & $21.497231890027(7)$ \\
\hline Spin period derivative, $\dot{P}\left(10^{-18}\right)$ & $2.6303(7)$ \\
\hline Timing epoch (MJD) & 57701 \\
\hline Dispersion measure, DM $\left(\mathrm{pc} \mathrm{cm}^{-3}\right)$ & $378.203(2)$ \\
\hline Binary model & $\mathrm{DDH}$ \\
\hline Orbital period, $P_{\mathrm{b}}(\mathrm{d})$ & $0.18353783587(5)$ \\
\hline Eccentricity, $e$ & $0.6058142(10)$ \\
\hline Projected semimajor axis, $x$ (lt-s) & $2.237805(5)$ \\
\hline Epoch of periastron, $T_{0}$ (MJD) & $57700.92599420(5)$ \\
\hline Longitude of periastron, $\omega\left({ }^{\circ}\right)$ & $279.3409(4)$ \\
\hline Rate of periastron advance, $\dot{\omega}\left({ }^{\circ} \mathrm{yr}^{-1}\right)$ & $10.3651(2)$ \\
\hline Einstein delay, $\gamma(\mathrm{ms})$ & $3.587(12)$ \\
\hline Orbital period derivative, $\dot{P}_{\mathrm{b}}\left(10^{-12}\right)$ & $-5.3(2)$ \\
\hline Orthometric amplitude, $h_{3}(\mu \mathrm{s})$ & $4.6(7)$ \\
\hline Orthometric ratio, $\varsigma$ & $0.90(3)$ \\
\hline Mass function, $f\left(\mathrm{M}_{\odot}\right)$ & $0.35718891(2)$ \\
\hline Total system mass, $M\left(\mathrm{M}_{\odot}\right)$ & $2.73295(9)^{\dagger}$ \\
\hline Pulsar mass, $m_{\mathrm{p}}\left(\mathrm{M}_{\odot}\right)$ & $1.3384(9)^{\dagger}$ \\
\hline Companion mass, $m_{\mathrm{c}}\left(\mathrm{M}_{\odot}\right)$ & $1.3946(9)^{\dagger}$ \\
\hline Inclination angle, $i\left(^{\circ}\right)$ & $84.0_{-0.3}^{+0.4}$ or $96.0_{-0.4}^{+0.3 \dagger}$ \\
\hline Flux density at $1.4 \mathrm{GHz}, S_{1400}(\mathrm{mJy})$ & $0.25(4)$ \\
\hline \multirow[t]{2}{*}{ DM distance, $d(\mathrm{kpc})$} & $7.4(\mathrm{NE} 2001)$ \\
\hline & 19.6 (YMW16) \\
\hline Surface magnetic field, $B_{\text {surf }}\left(10^{9} \mathrm{G}\right)$ & 7.61 \\
\hline Characteristic age, $\tau_{\mathrm{c}}(\mathrm{Myr})$ & 130 \\
\hline Spin-down luminosity, $\dot{E}\left(10^{30} \mathrm{ergs} \mathrm{s}^{-1}\right)$ & 10500 \\
\hline Time units & TCB \\
\hline Solar system ephemeris & DE421 \\
\hline RMS residual $(\mu \mathrm{s})$ & 36 \\
\hline
\end{tabular}

$\dagger$ Parameters derived according to the DDGR model.

$7.61 \times 10^{9} \mathrm{G}$, indicating that the pulsar has been partially recycled. Five PK parameters, including the rate of periastron advance $\dot{\omega}$, Einstein delay $\gamma$, orbital period derivative $\dot{P}_{\mathrm{b}}$ and orthometric Shapiro parameters $h_{3}$ and $\varsigma$, have been measured significantly. Using the DDGR model (Taylor 1987; Taylor \& Weisberg 1989), which assumes the correctness of GR, we derive the total system mass $M=2.73295(9) \mathrm{M}_{\odot}$ and the separate masses of the pulsar $\left(m_{\mathrm{p}}=1.3384(9) \mathrm{M}_{\odot}\right)$ and its companion $\left(m_{\mathrm{c}}=1.3946(9) \mathrm{M}_{\odot}\right)$. These masses, along with the high eccentricity and an implied second supernova (see Section 4) indicate that the system is a DNS. From $m_{\mathrm{p}}, m_{\mathrm{c}}$ and the mass function we can further infer an inclination angle of $i=84.0_{-0.3}^{+0.4}{ }^{\circ}$ (or $96.0_{-0.4}^{+0.3 \circ}$, when accounting for the $i \leftrightarrow 180^{\circ}-i$ ambiguity of the mass function), i.e., the orbit appears to be close to edge-on.

Fig. 2 shows the constraints on the NS masses derived from the measured PK parameters under the assumption of GR. By using the intersection of $\dot{\omega}$ and $\gamma$ to fix the two NS masses, we can derive three new tests of GR from the remaining PK parameters. Based upon the $\dot{\omega}-\gamma$ mass solu- 


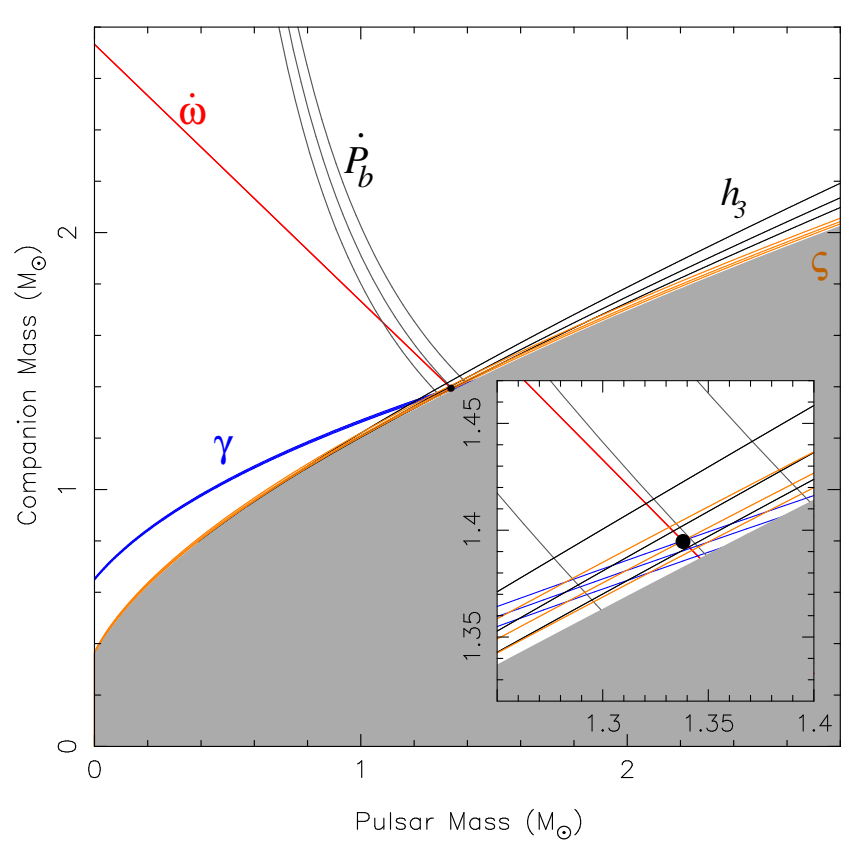

Figure 2. Mass-mass diagram for PSR J1757-1854. Shown are the mass constraints imposed under GR by each PK parameter, along with their 1- $\sigma$ error bars. A zoomed view of the region of intersection is shown in the inset, with the black dot indicating the DDGR masses. The grey region is excluded by orbital geometry.

tion, GR predicts an orbital decay due to GW damping of $\dot{P}_{\mathrm{b}}=-5.2747(6) \times 10^{-12}$, which the measured value of $\dot{P}_{\mathrm{b}}$ agrees with to within a relative uncertainty of only $5 \%$. For $\varsigma$ and $h_{3}$, both observed values are within 1- $\sigma$ agreement of their GR predicted values $\left(0.92_{-0.025}^{+0.040}\right.$ and $5.37_{-0.40}^{+0.72} \mu$ s respectively), indicating that GR passes all three tests.

PSR J1757-1854 exceeds many of the relativistic qualities of previous binary pulsars, setting records (among others) for the closest binary separation at periastron $\left(0.749 \mathrm{R}_{\odot}\right)$ and the highest relative velocity $\left(1060 \mathrm{~km} \mathrm{~s}^{-1}\right)$ at periastron. It also shows the strongest effects of GW damping yet seen in a relativistic pulsar binary, displaying the highest value of $\dot{P}_{\mathrm{b}}$ as well as the highest value of $\dot{P}_{\mathrm{b}} / P_{\mathrm{b}}=-3.33 \times 10^{-16} \mathrm{~s}^{-1}$, the leading-order term in the cumulative shift in periastron time (see e.g. Taylor \& Weisberg 1982). This results in an inferred merger time of $76 \mathrm{Myr}$. Hence, PSR J1757-1854 can be seen to probe a relativistic parameter space not yet explored by previous binary pulsars.

\subsection{Searches for the companion NS}

All GBT observations were recorded in coherently dedispersed search mode, with two methods used to search for pulsations from the companion NS. The first method involved breaking each de-dispersed time series into $\sim 30$ min segments, before performing a matched-filter acceleration search using the PRESTO program ACCELSEARCH with a filter width of $z_{\max }=50$ (where $z$ is the number of Fourier bins drifted by an accelerating pulsar). The second method (described in Martinez et al. 2015) involved resampling each time series in order to deconvolve the orbital motion of the companion NS as anticipated from the measured values of $m_{\mathrm{c}}$ and $m_{\mathrm{p}}$, before applying a PRESTO-based periodicity search. Neither method detected pulsations from the companion. As precession may move the beam of the companion into the line-of-sight in the future, we will continue to record search-mode data so as to repeat these studies regularly.

\section{EVOLUTIONARY HISTORY}

PSR J1757-1854 is remarkable given its combination of a short orbital period, a large eccentricity, a relatively massive young NS companion (more massive than the recycled pulsar, a property shared with only one other published DNS system, PSR B1534+12, Stairs et al. 2002), and a fast spinning recycled pulsar. The fast spin is expected for such a short orbital period DNS system, since in tight systems the recycling of the first-born NS is most efficient (see Case BB mass transfer modelling in Tauris et al. 2015, 2017).

Both the large eccentricity and the relatively massive young NS hint that a large kick is likely to have been imparted on the young NS at birth; see e.g. the mass-kick correlation suggested by Tauris et al. (2017). Indeed the two DNS systems B1913+16 and B1534+12, which also have relatively massive young NS companions, were shown to have experienced kicks of $\sim 200-400 \mathrm{~km} \mathrm{~s}^{-1}$.

To test this hypothesis for PSR J1757-1854, we performed Monte Carlo simulations of the kinematic effects of the second supernova following the method outlined in Tauris et al. (2017). As expected, we find that a large kick is most likely at work for this system (the broad distribution of solutions peaks at a value near $\left.400 \mathrm{~km} \mathrm{~s}^{-1}\right)$. For the mass of the exploding star, we find solutions from less than $2 \mathrm{M}_{\odot}$ and up to our maximum input limit of $7 \mathrm{M}_{\odot}$. However, the distribution peaks at the smallest value, supporting the idea of an ultra-stripped star exploding (Tauris et al. 2013, 2015).

\section{FUTURE PROSPECTS}

The simulations described in Section 4 also produce a systemic 3D velocity distribution for PSR J1757-1854, which peaks at a value of the order $200 \mathrm{~km} \mathrm{~s}^{-1}$. Assuming a representative velocity in the plane of the sky of $150 \mathrm{~km} \mathrm{~s}^{-1}$ and the NE2001 DM distance of 7.4-kpc (see Table 2) produces a predicted proper motion of 4.3 mas $\mathrm{yr}^{-1}$. Furthermore, for the recycled pulsar we also obtain a distribution of misalignment angles between the spin vector of the pulsar and the orbital angular momentum, with a median value of $\sim 25^{\circ}$.

Consequentially, PSR J1757-1854 is also expected to allow for future measurements of Lense-Thirring precession. Due to the large estimated distance to the pulsar, which suggests that we will not be able to correct for extrinsic acceleration effects sufficiently, we expect to be unable to employ the $\dot{\omega}-\dot{P}_{\mathrm{b}}$ measurement technique used on PSR J0737-3039 (Kehl et al. 2016). However, the likelihood of a significant misalignment angle allows an alternate test using the contribution of Lense-Thirring precession to the rate of change of the projected semi-major axis, given by

$\dot{x}_{\mathrm{LT}}=x \cot i\left(\frac{\mathrm{d} i}{\mathrm{~d} t}\right)_{\mathrm{LT}}$,

where $(\mathrm{d} i / \mathrm{d} t)_{\mathrm{LT}}$ is given by Eq. 3.27 in Damour \& Taylor 
(1992). Adopting a typically-assumed pulsar moment of inertia of $I=1.2 \times 10^{45} \mathrm{~g} \mathrm{~cm}^{2}$ (Lattimer \& Schutz 2005) and neglecting the likely slower-spinning companion NS, we calculate that $\left|\dot{x}_{\mathrm{LT}}\right|$ could be as large as $1.9 \times 10^{-14} \mathrm{lt}-\mathrm{s} \mathrm{s}^{-1}$. Based upon a continuation of our current timing setup, and assuming additional MeerKAT observations consisting of one orbit/month commencing in mid-2018, we predict a future measurement of $\dot{x}_{\mathrm{LT}}$ to within $3 \sigma$ in $\sim 8-9$ years. The corresponding (geodetic) spin precession of the pulsar (expected to be $\sim 3.1^{\circ} \mathrm{yr}^{-1}$ ) is expected to cause changes in the pulse profile and polarisation, which may allow a determination of the pulsar's spin orientation (see e.g. Kramer 1998).

PSR J1757-1854 is also an ideal system for measuring the PK parameter $\delta_{\theta}$, which describes the relativistic deformation of the elliptical orbit (Damour \& Deruelle 1985). To date, $\delta_{\theta}$ has been measured only in PSR B1913+16 (Weisberg \& Huang 2016) and PSR J0737-3039 (Kramer et al., in prep.), in both cases with low significance. As described in Damour \& Deruelle (1986), the timing residual contribution of $\delta_{\theta}$ can be characterised by

$\Delta_{\delta_{\theta}} \simeq-\delta_{\theta} \frac{e^{2}}{\sqrt{1-e^{2}}} x \cos \omega \sin u$,

where $u$ is the eccentric anomaly. The strong dependence of $\Delta_{\delta_{\theta}}$ on $e$ implies that PSR J1757-1854 (along with other high-e relativistic binaries such as PSRs B1913+16 and B2127+11C) will show the strongest timing effects due to $\delta_{\theta}$. However, Equation 2 also indicates that a measurement of $\delta_{\theta}$ requires a significant change in $\omega$ in order to separate the residual effect of $\delta_{\theta}$ from that of $\gamma$ (for which $\Delta_{\gamma}=\gamma \sin u$ ). With its high $\dot{\omega} \simeq 10.37^{\circ} \mathrm{yr}^{-1}$, PSR J1757-1854 is therefore uniquely positioned to allow for a future measurement of $\delta_{\theta}$ within a comparatively-short timeframe. Based on the same timing considerations as outlined for $\dot{x}_{\mathrm{LT}}$, we predict a 3- $\sigma$ measurement of $\delta_{\theta}$ will be possible within $\sim 7-8$ years.

Finally, as noted in Section 3.2, PSR J1757-1854 has the largest observed $\dot{P}_{\mathrm{b}}$ and the largest shift in periastron time due to GW emission of any known binary pulsar. This promises a further high-precision test of GR's quadrupole formula for $\mathrm{GW}$ damping, as conducted previously with PSR B1913+16 (Weisberg \& Huang 2016) and PSR J0737-3039 (Kramer et al. 2006; Kramer 2016). Timing simulations indicate a test precision of $<1 \%$ in only $\sim 5$ years. Taking the Galactic potential of McMillan (2017) and our previous systemic velocity estimates, we anticipate that the uncertainties on the individual estimates of the distance to PSR J1757-1854 (see Cordes \& Lazio 2002; Yao et al. 2017) will limit this test to within a few tenths of a percent.

\section{ACKNOWLEDGEMENTS}

The Parkes Observatory is part of the Australia Telescope National Facility which is funded by the Australian Government for operation as a National Facility managed by CSIRO. The Green Bank Observatory is a facility of the National Science Foundation operated under cooperative agreement by Associated Universities, Inc. Pulsar research at the Jodrell Bank Centre for Astrophysics and the observations using the Lovell Telescope are supported by a consolidated grant from the STFC in the UK. This work is also based on observations with the 100-m telescope of the Max-Planck-Institut für Radioastronomie at Effelsberg. This work is supported by the ARC Centres of Excellence CE110001020 (CAASTRO) and CE170100004 (OzGrav). Survey processing was conducted in association with CAASTRO at the Australian National Computational Infrastructure high-performance computing centre at the Australian National University. The authors wish to thank Marina Berezina, Eleni Graikou, Alex Kraus and Laura Spitler for their assistance with observations at Effelsberg, along with Natalia Lewandowska and Ryan Lynch for their assistance with observations at the GBT. We also thank West Virginia University for its financial support of GBT operations, which enabled some of the observations for this project. AC acknowledges the support of both the International Max Planck Research School for Astronomy and Astrophysics at the Universities of Bonn and Cologne, and the Bonn-Cologne Graduate School of Physics and Astronomy. PCCF and AR gratefully acknowledge financial support by the European Research Council for the ERC Starting grant BEACON under contract No. 279702, and continued support from the Max Planck Society. MK, RK and RPE gratefully acknowledge support from ERC Synergy Grant 'BlackHoleCam' Grant Agreement Number 610058. MAM was supported by NSF award number AST-1517003. DRL was supported by NSF award number OIA-1458952.

\section{REFERENCES}

Abbott B. P., et al., 2016, Phys. Rev. Lett., 116, 061102

Abbott B. P., et al., 2017, Phys. Rev. Lett., 119, 161101

Bassa C. G., et al., 2016, MNRAS, 456, 2196

Belczynski K., Kalogera V., Bulik T., 2002, ApJ, 572, 407

Breton R. P., et al., 2008, Science, 321, 104

Burgay M., et al., 2003, Nature, 426, 531

Cordes J. M., Lazio T. J. W., 2002, preprint, (arXiv:astro$\mathrm{ph} / 0207156)$

Damour T., Deruelle N., 1985, Ann. Inst. Henri Poincaré Phys. Théor., No. 1, 43, 107

Damour T., Deruelle N., 1986, Ann. Inst. Henri Poincaré Phys. Théor., No. 3, 44, 263

Damour T., Taylor J. H., 1992, Phys. Rev. D, 45, 1840

DuPlain R., Ransom S., Demorest P., Brandt P., Ford J., Shelton A. L., 2008, in Advanced Software and Control for Astronomy II. p. $70191 \mathrm{D}$, doi:10.1117/12.790003

Eatough R. P., Kramer M., Lyne A. G., Keith M. J., 2013, MNRAS, 431, 292

Freire P. C. C., Wex N., 2010, MNRAS, 409, 199

Freire P. C. C., et al., 2012, MNRAS, 423, 3328

Hobbs G. B., Edwards R. T., Manchester R. N., 2006, MNRAS, 369,655

Hotan A. W., van Straten W., Manchester R. N., 2004, Publ. Astron. Soc. Australia, 21, 302

Hulse R. A., Taylor J. H., 1975, ApJ, 195, L51

Johnston H. M., Kulkarni S. R., 1991, ApJ, 368, 504

Kehl M. S., Wex N., Kramer M., Liu K., 2016, preprint, (arXiv:1605.00408)

Keith M. J., et al., 2010, MNRAS, 409, 619

Kramer M., 1998, ApJ, 509, 856

Kramer M., 2016, International Journal of Modern Physics D, 25, 1630029

Kramer M., et al., 2006, Science, 314, 97

Lattimer J. M., Schutz B. F., 2005, ApJ, 629, 979

Lazarus P., Karuppusamy R., Graikou E., Caballero R. N., Cham- 
pion D. J., Lee K. J., Verbiest J. P. W., Kramer M., 2016, MNRAS, 458, 868

Lorimer D. R., Kramer M., 2004, Handbook of Pulsar Astronomy

Lyne A. G., et al., 2004, Science, 303, 1153

Manchester R. N., et al., 2001, MNRAS, 328, 17

Martinez J. G., et al., 2015, ApJ, 812, 143

McMillan P. J., 2017, MNRAS, 465, 76

Middleditch J., Kristian J., 1984, ApJ, 279, 157

Ng C., et al., 2015, MNRAS, 450, 2922

Ransom S. M., 2001, PhD thesis, Harvard University

Ransom S. M., Eikenberry S. S., Middleditch J., 2002, AJ, 124, 1788

Shao L., Sennett N., Buonanno A., Kramer M., Wex N., 2017, Physical Review X, 7, 041025

Stairs I. H., Thorsett S. E., Taylor J. H., Wolszczan A., 2002, ApJ, 581, 501

Staveley-Smith L., et al., 1996, Publ. Astron. Soc. Australia, 13, 243

Tauris T. M., Langer N., Moriya T. J., Podsiadlowski P., Yoon S.-C., Blinnikov S. I., 2013, ApJ, 778, L23

Tauris T. M., Langer N., Podsiadlowski P., 2015, MNRAS, 451, 2123

Tauris T. M., et al., 2017, ApJ, 846, 170

Taylor J. H., 1987, in MacCallum M. A. H., ed., General Relativity and Gravitation, Cambridge, UK: Cambridge University Press. pp 209-222

Taylor J. H., Weisberg J. M., 1982, ApJ, 253, 908

Taylor J. H., Weisberg J. M., 1989, ApJ, 345, 434

Weisberg J. M., Huang Y., 2016, ApJ, 829, 55

Wex N., 2014, in Kopeikin S., ed., Frontiers in Relativistic Celestial Mechanics, Volume 2: Applications and Experiments. De Gruyter, Berlin/Boston, pp 35-98 (arXiv: 1402.5594)

Yao J. M., Manchester R. N., Wang N., 2017, ApJ, 835, 29

van Straten W., Bailes M., 2011, Publ. Astron. Soc. Australia, 28,1

This paper has been typeset from a $\mathrm{T}_{\mathrm{E}} \mathrm{X} / \mathrm{LAT}_{\mathrm{E}} \mathrm{X}$ file prepared by the author. 